\title{
Editorial
}

Rüstungskontrolle, als Instrument der Konfliktreduzierung und Friedensförderung von der Staatenwelt nie besonders geliebt, hat derzeit keine Konjunktur, wofür nicht zuletzt die neueren Trends in der amerikanischen Außenpolitik verantwortlich sind. Dennoch ist sie ein Gebot der Vernunft. Die vorliegende Ausgabe von $\mathrm{S}+\mathrm{F}$ widmet sich im Schwerpunkt diesem vernachlässigten Politikfeld.

Rolf Mützenich und Matthias Z. Karádi stellen die Frage nach der Zukunft der Rüstungskontrolle und insbes. der Perspektiven ihrer Implementierung und Verifikation. Sie verdeutlichen die Schlüsselrolle der USA auf diesem Politikfeld wie ihr Abdriften ins rüstungskontrollpolitische Abseits und unterbreiten Vorschläge zur Rettung eines zentralen Instruments der internationalen Sicherheitspolitik.

Dem Problem der regionalen Rüstungskontrolle in Asien widmet sich der Beitrag von Christopher Daase und Susanne Feske. Er zeigt die regionsspezifischen Merkmale der dortigen Rüstungsdynamiken wie der rüstungskontrollpolitischen Ansätze auf und gibt Auskunft über die Erfolgsbedingungen kooperativer Rüstungssteuerung.

Politische Handlungsmöglichkeiten zur Kontrolle biologischer Waffen untersuchen Oliver Meier und Jan van Aken: Die Krise des internationalen Biowaffenverbots kann nur durch ein intensiviertes Engagement abrüstungsfreundlicher Staaten und der weltweiten Zivilgesellschaften überwunden werden.

Außerhalb des Themenschwerpunkts stellen Erwin Müller und Patricia Schneider optimierte Modelle der Friedenssicherung durch internationale Rechtsprechung und Rechtsdurchsetzung vor: Institutionen und Mechanismen der friedlichen Streitbeilegung durch Mediation, Arbitration und Jurisdiktion, die eine Zivilisierung des Konfliktaustrags gewährleisten sollen.

Die ebenso mannigfaltigen wie unübersichtlichen Konflikte in Indonesien dokumentiert der Aufsatz von Kathryn Tätzsch. Ihre Ursachen finden sich u.a. in religiösen Zerwürfnissen, sozialen Gegensätzen, politischen Partizipationsdefiziten und Autonomiebestrebungen.

Christoph Busch und Markus Birzer thematisieren die Internet-Strategien des rechtsextremistischen Spektrums zur Durchsetzung seiner Ziele. Die Analyse zeigt, dass diese Kräfte sich auch im »virtuellen Raum« neue Optionen der Einflussnahme erschließen.

Die nächste Ausgabe von $\mathrm{S}+\mathrm{F}$ wird sich wiederum dem Thema Rüstungskontrolle widmen.

\section{Die Zukunft der Rüstungskontrolle: Perspektiven bei der Implementierung und Verifikation*}

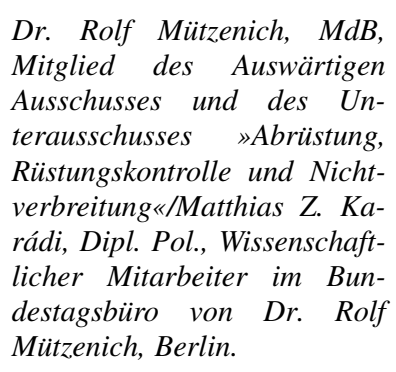

\section{Einleitung}

Die Rüstungskontrolle befindet sich - je nach Betrachtungsweise - in der Krise, der Stagnation oder ist bereits sanft verschieden. Nach dem Ende des Kalten Krieges steht das Konzept der Rüstungskontrolle heute vor einer doppelten Herausforderung:

Es muss sowohl den strukturellen Veränderungen des internationalen Systems nach 1989 als auch der technologischen Dynamik des Informationszeitalters angepasst werden. Einerseits distanzieren sich die maßgeblichen Entscheidungsträger

* Unter Mitarbeit von Benkis Skrijelj. in den USA vom Konzept der Abrüstung und Rüstungskontrolle, andererseits sind die bestimmenden Triebkräfte der Informations- und Kommunikationstechnologie heute in erster Linie im kommerziellen Bereich zu finden. Vor allem im Bereich der Informationstechnologien, die heutzutage Eingang in praktisch alle modernen Waffensysteme gefunden haben, verwischt damit zunehmend die Grenze zwischen militärischen und zivilen Systemen. Auch für die Rüstungskontrolle hat somit das postmoderne Zeitalter begonnen. ${ }^{1}$

Zudem sind im letzten Jahrzehnt traditionelle Annahmen über die in Rüstungskontrollfragen relevanten Akteure und Gegnerschaften weitgehend hinfällig geworden. Die Konflikt-

1 Vgl. auch Bericht der Bundesregierung zum Stand der Bemühungen um Rüstungskontrolle, Abrüstung und Nichtverbreitung sowie über die Entwicklung der Streitkräftepotenziale (Jahresabrüstungsbericht 2002), Drucksache 15/1104, Berlin, 02.06.2003. 
linien verlaufen nicht mehr zwischen zwei Weltanschauungen, sondern sind abhängig von Situationsbedingungen, die schnell wechseln können. Auch profitierte die Rüstungskontrolle während des Kalten Krieges von der Akteursstruktur, also der Teilung in zwei formale Allianzen, während es in vielen aktuellen Konflikten zunehmend schwieriger ist, die relevanten Akteure zu identifizieren. Zwar hat die Gefahr eines nuklearen Weltkrieges abgenommen, gleichzeitig treten aber an die Stelle dieser klar zu bestimmenden Bedrohung bisher unbekannte Gefahren für die internationale Sicherheit: schwache und instabile Staaten, die mit Massenvernichtungswaffen ausgerüstet sind, oder nicht-staatliche Akteure, die immer mehr an Bedeutung gewinnen. Als neue Herausforderung für die Rüstungskontrollbemühungen erweisen sich zunehmend die schwieriger werdende Aufrechterhaltung des staatlichen Gewaltmonopols, die Verminderung der Zerstörungswirkung von Waffen und die Reduzierung ihrer Kosten.

\section{Rüstungskontrolle zu Beginn des 21. Jahrhunderts}

Die Abrüstungsdekade der neunziger Jahre, scheint endgültig beendet zu sein. Zu Beginn des 21. Jahrhunderts bahnt sich ein neues Wettrüsten an. Während die US-Regierung unverhohlen ihr Desinteresse an Rüstungskontrollverhandlungen zeigt und mit ihrer Politik auf militärische Stärke und Suprematie setzt, scheinen die Europäer mittlerweile Abrüstungsinitiativen weitgehend aufgegeben $\mathrm{zu}$ haben. Sogar bereits unterzeichnete Abkommen, wie der KSE-IIVertrag, können nicht in Kraft treten, weil Deutschland das entsprechende Ratifikationsverfahren mit Rücksicht auf neue Bedenken der USA nicht betreibt. Dabei erfordern die neuen sicherheitspolitischen Herausforderungen gerade auch Antworten im Bereich der Rüstungskontrolle. Hierzu gehören vertrauens- und sicherheitsbildende Maßnahmen, Verifikation sowie Nichtweiterverbreitung und Abrüstung. Ganz oben auf der Agenda steht die Verhinderung der Proliferation von Massenvernichtungswaffen, aber auch des ungehinderten Transfers von konventionellen Waffen und Kleinwaffen. Nach wie vor gilt es, Rüstung zu begrenzen und konsequent abzurüsten, was nicht für die Sicherung des Friedens benötigt wird. Leider scheinen die USA und ihre Verbündeten als einzige Lehre aus dem 11. September den Ausbau der militärischen Suprematie verbunden mit der Führung von Präventivkriegen gezogen zu haben.

Trotz dieser krisenhaften Entwicklungen sollten auch nicht die positiven Entwicklungen und Erfolge der neunziger Jahre vergessen werden: 1991 ist Südafrika dem Nuklearen Nichtverbreitungsvertrag (NPT) beigetreten und hat seine bereits produzierten Nuklearwaffen vollständig vernichtet. Die drei »nuklearen Nachfolgestaaten « der Sowjetunion - die Ukraine, Belarus und Kasachstan - haben 1994 auf ihre Nuklearwaffen verzichtet und sind dem NPT beigetreten. Dieser wurde 1995 unbefristet verlängert, ihm gehören mittlerweile 187 Staaten an, also alle Staaten außer Indien, Pakistan, Israel und Kuba bis auf Kuba allerdings alles de facto Nuklearmächte. 1996 einigte man sich nach jahrzehntelangen Verhandlungen über einen Vertrag über einen umfassenden nuklearen Teststopp (CTBT). Bedauerlich ist, dass die USA dieses wichtige Abkommen noch nicht ratifiziert haben. Offiziell werden Bedenken zur Verifizierbarkeit des Vertrages angeführt. Im
Kern ist die Ablehnung allerdings das Spiegelbild einer neuen Sicherheitsdoktrin der USA, welche besagt, alle Maßnahmen zu unterlassen, die die Handlungsmöglichkeiten der Weltmacht einschränken könnten. Im Jahre 1997 trat das Übereinkommen über das weltweite Verbot chemischer Waffen (CWÜ) in Kraft, das bislang von 174 Staaten unterzeichnet und von 145 ratifiziert wurde. Der erweiterte KSE-Vertrag vom November 1999 senkt das Streitkräfteniveau in Europa um ca. 11.000 Waffensysteme. Erweiterte Transparenz- und Verifikationsmaßnahmen in Bezug auf Dislozierung, Aufwuchs, Verlegung und Reduzierung von Truppen ergänzen die neuen Regelungen und unterstreichen die Schlüsselrolle des KSE-Vertrags für die europäische Sicherheit. Auf der Überprüfungskonferenz des NPT gaben die fünf offiziellen Atommächte 2000 eine Erklärung ab, die vollständige Abschaffung ihrer Arsenale bis zur vollständigen nuklearen Abrüstung zu betreiben. Am 24. Mai 2002 unterzeichneten die USA und Russland einen neuen Abrüstungsvertrag namens SORT/Strategic Offensive Reductions Treaty. Danach sollen die strategischen Nuklearwaffen von derzeit je rund 6.000 auf 1.700 bis 2.200 Sprengköpfe reduziert werden. Problematisch ist die zeitliche Begrenzung des Vertrages und die fehlende Verifikation. Weiterhin ist positiv anzumerken, dass in jüngster Zeit zumindest in offiziellen Verlautbarungen Maßnahmen der Rüstungskontrolle wieder ein politischer Stellenwert beigemessen wurde. So haben sich die USA und die EU auf gemeinsame Schritte verständigt, um die Verbreitung von Massenvernichtungswaffen zu unterbinden. Die Staats- und Regierungschefs der EU haben am 20. Juni 2003 beschlossen, bestehende Nichtverbreitungsverträge zu stärken, Exportkontrollen zu intensivieren, die internationale Zusammenarbeit auszubauen und den politischen Dialog mit anderen Ländern zu vertiefen. Die G8 haben eine gemeinsame Erklärung verabschiedet, worin die Verträge gegen die Verbreitung von Atom-, Chemie- und Bio-Waffen ausdrücklich gewürdigt werden.

Daneben ist allerdings auch eine Vielzahl von negativen Entwicklungen und rüstungskontrollpolitischen Rückschlägen zu beklagen: Eine Reihe von wichtigen Verträgen ist nicht ratifiziert und daher nicht oder nur teilweise implementiert worden: der Open-Skies-Vertrag von 1992, der amerikanischrussische START-II-Vertrag von 1993 über die Reduzierung der strategischen Nuklearwaffen oder der Vertrag über einen Umfassenden Nuklearteststopp (CTBT) von 1996. Andere Verhandlungen haben trotz jahrelanger Vorbereitungen noch nicht begonnen oder stecken in der Sackgasse, wie die Verhandlungen über ein Verbot der Militarisierung des Weltraums oder der Vertrag über einen Produktionsstopp für waffenfähiges Spaltmaterial (Cut off). Die Blockade der Genfer Abrüstungskonferenz geht mittlerweile ins siebte Jahr. Mit Nordkorea ist das erste Land in der Geschichte des Atomwaffen-Sperrvertrags (NPT) aus dem Vertrag ausgestiegen. Die nukleare Schwellenmacht Nordkorea hatte den Vertrag 1985 unterzeichnet. Pjöngjang beendete zudem seine Bindung an das Atom-Sicherheitsabkommen mit der Internationalen Atomenergie-Organisation (IAEO). Nordkorea rechtfertigte den Austritt aus dem Atomwaffensperrvertrag damit, dass die USA einseitig ihre Verpflichtungen zur Beendigung der nuklearen Bedrohung und zum Verzicht auf Feindseligkeiten gegenüber Nordkorea aufgegeben hätten. Pjöngjang unterstrich zudem den Bedarf an Energie, da 
Washington die nach dem Abkommen von 1994 zugesagten Öllieferungen gestoppt habe. Die USA hatten damit auf das Eingeständnis Nordkoreas vom Oktober 2002 reagiert, ein Programm zur Urananreicherung zu betreiben. Nordkorea hatte schon während des Atomkonflikts 1993 die Absicht zum Rückzug aus dem Sperrvertrag erklärt, jedoch ein Moratorium verhängt. $^{2}$

Zweifelsohne bedarf die Rüstungskontrolle dringend neuer Impulse, um den Abrüstungsprozess wieder in Gang zu bringen. Dies wird alles andere als leicht werden. So ist es immer einfacher über die Abrüstung anderer $\mathrm{zu}$ reden, als über die eigene: Es führt leider kein Weg an der Erkenntnis vorbei, dass die USA - ebenso wie die meisten NATO-Mitglieder - derzeit ihr Heil weniger in den Themenbereichen Abrüstung und Rüstungskontrolle, als vielmehr in der Schaffung von gesteigerten und verbesserten militärischen Interventionsfähigkeiten sehen. Die Beschlüsse der NATO und des EU-Konvents zielen jedenfalls eindeutig in diese Richtung. Nichtverbreitung, Abrüstung und Rüstungskontrolle müssen deshalb wieder verstärkt als wesentlicher Bestandteil einer europäischen Sicherheitspolitik verstanden werden. ${ }^{3}$ Angesichts der globalen sicherheitspolitischen Herausforderungen brauchen wir dringend neue Initiativen und verstärkte transatlantische Zusammenarbeit in den Bereichen Rüstungskontrolle und Nichtverbreitung. Dabei müssen sowohl die vorhandenen Abrüstungs- und Nichtverbreitungsregime gestärkt und verbessert, als auch die Entwicklung effizienterer Kontroll- und Sanktionsmechanismen in Angriff genommen werden. Dies ist die einzige Möglichkeit, um auch die USA an das Konzept der Rüstungskontrolle wieder heranzuführen. Die Weltmacht wird diesem Instrumentarium um so mehr Beachtung schenken müssen, wie die Initiativen und Aktionen für eine vermeintlich größere Sicherheit (Prävention, Präemption, Raketenabwehr) an der Realität scheitern. In diesem Augenblick könnte das Konzept einer »robusten Rüstungskontrolle« die USA auf den Weg einer multilateralen Arbeitsteilung zurückführen.

\section{Technologische Entwicklung und Verifikation}

Vor dem Hintergrund der in der Folge des Zusammenbruchs der Sowjetunion aufgetretenen Veränderungen, ist die schnelle Verbreitung von Hoch-Technologie über alle nationalen Grenzen hinweg eine der wichtigsten Entwicklungen im Bereich der Sicherheit. Weltweit kommt es zu einer tiefgreifenden technologischen Modernisierung der Streitkräfte, vielfach werden durch diese neue qualitative Aufrüstung vorherige quantitative Abrüstungsmaßnahmen mehr als kompensiert. An die Stelle der zahlenmäßigen Erhöhung der Bestände an Waffen und Personen ist heute die technologisch gestuitzte Verbesserung und Modernisierung getreten: das qualitative Wettrüsten. Im Umgang mit diesem neuen Wettrüsten steht die Rüstungskontrolle vor neuen Problemstellungen, die sich

2 Vgl. hierzu Sebastian Harnisch, Nordkoreas nukleare Waffenprogramme: Entstehung, Fähigkeiten und die internationalen Bemühungen um ihre Eindämmung, in: Österreichische Militärische Zeitschrift/ÖMZ 2/2003, S. 149-162.

3 Vgl. hierzu das vom Europäischen Rat der Außenminister verabschiedete Dokument $\gg$ Basic Principles for an EU Strategy against Proliferation of Weapons of Mass Destruction«, Luxemburg, 16. Juni 2003. zum Einen aus den Besonderheiten der Technologien und ihrer Entwicklung ableiten, und zum Anderen aus der bisherigen Tradition der Rüstungskontrolle, sich auf quantitative Beschränkungen zu konzentrieren, resultieren.

Der Rüstungskontrollprozess hat die Fortschritte im Bereich der Verifikation mit vorbereitet. Dies begann mit der Einrichtung der Standing Consultative Commission im Rahmen der SALT-Vereinbarungen und setzte sich in den Verträgen der siebzigerr Jahre fort, in denen u.a. Satellitenbeobachtung als völkerrechtlich zulässige Form der Verifikation durch technische Mittel anerkannt wurde. Darüber hinaus wurden gemeinsame Kommissionen, Vorankündigungen militärischer Maßnahmen bis hin zu Vor-Ort-Inspektionen als Verifikationselemente in diverse Verträge eingeführt. Um die Inspektionsregime, die in diversen Rüstungskontrollverträgen vorgesehen sind, zu verbessern, werden neben Erst- und Routineinspektionen auch zunehmend Verdachtsinspektionen und nicht angemeldete Inspektionen durchgeführt.

Während zu Zeiten des Kalten Krieges Rüstungskontrollabkommen in erster Linie bzw. ausschließlich durch nationale Regierungen überprüft und verifiziert wurden, wird seit dem Ende des Ost-West-Konflikts die Verifikation verstärkt von spezialisierten internationalen Behörden wie der Internationalen Atomenergiebehörde (IAEO) in Wien, der Organisation für das Verbot chemischer Waffen $(\mathrm{OVCW})^{4}$ mit Sitz in Den Haag und der im Aufbau befindlichen Atomteststopporganisation (CTBTO) überwacht. Vorbildhaft ist insbesondere das Zentrum für Verifikationsaufgaben der Bundeswehr.

Verifiaktion wird hier verstanden als Sammlung und Analyse von Informationen, um die Vertragstreue von Mitgliedstaaten eines internationalen Abkommens $\mathrm{zu}$ überprüfen. ${ }^{5}$ Verifikation soll dazu beitragen, relevante Vertragsverletzungen rechtzeitig aufzudecken, von solchen Verletzungen abzuschrecken sowie einen Kooperationsrahmen zwischen den Vertragsparteien aufzubauen, der auf gegenseitigem Vertrauen beruht. 6 Oder in den Worten Manfred Efingers: »Das Wort Verifikation kann in der deutschen Sprache die Bedeutungen Bewahrheitung und Beurkundung tragen. Für das Verb >verifizieren< wird dagegen zumeist die Bedeutung über- bzw. nachprüfen angegeben. In diesem Zusammenhang ist es sinnvoll, sich an die Bedeutungszuschreibung des Verbs zu halten, denn es geht darum, die Einhaltung von Normen und Regeln, die in Abrüstungs- bzw. Rüstungssteuerungsabkommen festgelegt sind, zu überprüfen. «7

Teil der bestehenden Verifiaktionsregime sind regelmäßige und stichprobenartige Kontrollen und Vor-Ort-Inspektionen. Um die Verbreitung von Massenvernichtungswaffen wirksam $\mathrm{zu}$ kontrollieren und zu unterbinden, müssen Verifikationsregime eine weitgehend umfassende und möglichst lückenlose

4 Das CWÜ verbietet Entwicklung, Herstellung, Beschaffung, Besitz, Weitergabe sowie den Einsatz chemischer Waffen und sieht vor, dass zehn Jahre nach seinem Inkrafttreten alle im Besitz der Vertragsstaaten befindlichen chemischen Waffen samt den dazugehörigen Produktionsanlagen zu vernichten sind.

$5 \mathrm{Zu}$ den aktuellen Entwicklungen im Bereich der Verifikation vgl. Trevor Findlay/Oliver Meier (Hrsg.), Verification Yearbook 2001: The Verification Research, Training and Information Centre (VERTIC), London 2001.

6 So die Definition von Oliver Meier, Neue Verifikationskonzepte, i.E.

7 Manfred Efinger, Vertrauen ist gut, Kontrolle ist besser, Baden-Baden 1991, S. 64. 
Kontrolle bieten. Dies können sie zumeist nur bedingt leisten. Dies liegt nicht nur an den technologischen Schwierigkeiten, sondern auch und wesentlich an der nach wie vor zu geringen finanziellen Ausstattung von Verifikationsabkommen und -behörden. Der technische Fortschritt macht sich jedoch nicht nur bei der Entwicklung von neuen Waffensystemen, sondern auch im Bereich der Verifikationstechnologien bemerkbar. Vor allem im Bereich der Satellitenfernerkundung sind erhebliche Fortschritte gemacht worden. Die Infrarot-Technik bietet neue Möglichkeit für Aufklärung und Überprüfung.

Verifikation muss, wenn sie ernst genommen werden soll, wesentlich mehr sein als nur gut gemeint. M.a.W.: Sie muss mehr leisten als »nur« Datenaustausch und Vertrauensbildung. Verifikation bedeutet in diesem Sinne die unzweideutige und weitgehend fälschungssichere Überprüfung sicherheitspolitisch und technisch relevanter Sachverhalte. Bei Rüstungsgütern mit Dual use-Eigenschaften stößt diese Vorgabe schnell an die Grenze. Vor allem aufgrund der jüngsten Entwicklungen im Bereich der Informations- und Nanotechnologie ist es kaum mehr möglich, potenzielle Waffenverwendungen moderner Mikro- und Nanotechnologien bei internationalen Kontrollen ausreichend zu überprüfen. Durch die extreme Miniaturisierung sind solche Waffen (Sensoren, Navigationsgeräte, Kampfkraftverstärker etc.) relativ leicht $\mathrm{zu}$ tarnen und $\mathrm{zu}$ verstecken. ${ }^{8}$ Hier unterscheidet sich die Verifikationsproblematik deutlich von der bei der Kontrolle der Chemie- und Atomwaffenprogramme, die sich auf eine eng begrenzte Zahl von Einrichtungen und Materialien mit relativ klar zu kontrollierenden Betriebs- und Gebrauchseigenschaften beschränkt. Dennoch ist Verifikation in erster Linie ein politisches und weniger ein technisches Problem. ${ }^{9}$ Entscheidend für die Verbesserung und den Ausbau von Überprüfungs- , Kontroll- und Überwachungssystemen ist und bleibt der politische Wille der nationalen Regierungen.

\section{Die Rolle der USA}

Die gegenwärtige Krise der Rüstungskontrolle hängt wesentlich mit der Politik der USA zusammen. Es lässt sich feststellen, dass die Vereinigten Staaten kein bzw. immer weniger Vertrauen in die internationalen Kontrollregime haben, die sie als zunehmend lückenhaft und ineffizient empfinden. Folgt man den Argumenten der Mitglieder der BushAdministration, so muss sich die traditionelle Rüstungskontrolle in folgenden Bereichen in Frage stellen lassen:

- Beim Verbot von Raketenabwehr: Diese könne nicht mehr per se als destabilisierend angesehen werden; vielmehr könnten Raketenabwehrsysteme dazu dienen, neuen Gefährdungen zu begegnen, und außerdem eröffneten sie neue Optionen der Reduzierung von strategischen Angriffswaffen.

- Bei der Nützlichkeit komplexer Rüstungskontrollverträge: Hier sei zu fragen, ob es weiterhin Sinn mache, als Ergebnis von Rüstungskontrollverhandlungen in jedem

8 Vgl. hierzu Gebhard Geiger, Mikrotechnologische Herausforderungen der internationalen Sicherheit, SWP, Berlin, Juni 2003, S. 19-21.

9 Vgl. auch Angelika Brinkmann, Verifikation und (Rüstungs-)Kontrolle, Baden-Baden 1996
Fall umfängliche, Hunderte von Seiten umfassende Vertragswerke $\mathrm{zu}$ schaffen, die die Verhandlungen ebenso in die Länge zögen wie die Ratifikation. Es stelle sich zunehmend die Frage, ob informelle Vereinbarungen nicht nützlicher seien.

- Bei der Nützlichkeit von multilateralen Verhandlungen: Multilaterale Abrüstungs- und Rüstungskontrolldiplomatie sei ebenso überflüssig wie teuer, was man insbesondere an der Tätigkeit (oder Untätigkeit) der Abrüstungskonferenz und der UN-Abrüstungskommission sehe. Dies bedeute nicht, dass alle multilateralen Foren aufzugeben seien insbesondere nicht der NPT -, aber es zeige, dass Zweifel an der Befassung multilateraler Foren mit komplexen Rüstungskontroll- und Abrüstungsmaterien bestünden.

Offenbar glauben die USA mit zunehmender politischer und militärischer Dominanz, kleinere Staaten militärisch unter Kontrolle halten zu können, selbst wenn diese nach $\mathrm{Nu}$ klearwaffen oder anderen Massenvernichtungswaffen streben. Die Vereinigten Staaten wollen offensichtlich eine Form der Rüstungskontrolle, die Waffensysteme anderer Staaten begrenzt, aber den eigenen Handlungsspielraum für weltweite militärische Aktionen nicht einschränkt. Dies wird am deutlichsten bei den Planungen zur Raketenabwehr. Ein aus der Geschichte der Abrüstungspolitik bekanntes Sicherheitsdilemma tritt erneut auf: Sobald es eine Hegemonialmacht gibt oder militärische Macht asymmetrisch verteilt ist, kommt Rüstungskontrolle zum Stillstand. Die Überlegenen sehen keinen Anlass mehr, ihre Übermacht aufzugeben oder zu beschränken. Dies wiederum hat die fatale Folge, dass sich die Unterlegenen ihrerseits zur Aufrüstung genötigt sehen.

Der Krieg gegen den Irak wurde als Abrüstungskrieg angekündigt und geführt, auch wenn die Massenvernichtungswaffen, wegen derer er angeblich geführt wurde, bis heute nicht aufgetaucht sind. Das eigentliche Ziel des Dritten Golfkrieges dürfte jedoch der gewaltsame Sturz Saddam Husseins und die Umsetzung der neuen Sicherheitsdoktrin gewesen sein. Nachdem man die Massenvernichtungswaffen offensichtlich nicht finden konnte, rückt mittlerweile der Sturz eines verbrecherischen Regimes als offizielle Kriegsbegründung in den Vordergrund. Es bleibt jedoch ein Makel des Irakkrieges, dass die Führer der Kriegskoalition ihre Bürger und die der Welt getäuscht und in die Irre geführt haben. Darüber hinaus wachsen die Befürchtungen, der Krieg gegen den Irak sei nur der erste in einer ganzen Reihe von geplanten »Abrüstungskriegen«. Werden künftig unbotmäßige Regime, in denen Terroristen und/oder Massenvernichtungswaffen vermutet werden, präemptiv und präventiv mit Abrüstungs- oder Antiterrorkriegen überzogen werden $?^{10}$ Und stehen der Iran und Nordkorea als Nächstes auf der amerikanischen Tagesordnung? Hier besteht allerdings die begründete Hoffnung, dass es in einer konzertierten Aktion von EU und USA gelingen

10 Traditionell wird zwischen präemptiven und präventiven Militärschlägen hinsichtlich des Kriteriums der »unmittelbar bevorstehenden Bedrohung « unterschieden. Wird militärisch gegen eine unmittelbare und imminente Bedrohung vorgegangen, so spricht man von einem Präemptivschlag (Israels Überraschungsangriff während des Sechstagekriegs 1967 dient hier in der Regel als Beispiel); ist die Bedrohung "nur « mittelbar oder noch gar nicht gegeben, spricht man von militärischer Prävention (Hier gilt die Zerstörung des irakischen Osirak-Atomreaktors durch die israelische Luftwaffe im Juni 1981 als Paradebeispiel). 
kann, die Konflikte um die iranischen und nordkoreanischen Waffenprogramme friedlich auf dem Verhandlungswege zu lösen.

Die entscheidende Frage jedoch bleibt, was eine Rüstungskontrollpolitik leisten müsste, um Washington davon zu überzeugen, dass Prävention durch Rüstungskontrolle der Prävention durch Entwaffnungskriege allemal vorzuziehen ist. Sie müsste zweifelsohne effizienter, im Sinne von genauer und überprüfbarer, werden. Dies bedeutet in erster Linie die Entwicklung von klaren, wirksamen und überprüfbaren Verifikationsregimen. Verifikation wird somit zur Schlüsselfrage für künftige Rüstungskontrollabkommen, zumindest wenn diese die Zustimmung der USA erlangen sollen.

Sollten die Vereinigten Staaten hingegen ihre gegenwärtige Obstruktionspolitik fortsetzen, so müsste man auch verstärkt über Gegenstrategien nachdenken. Dies impliziert notfalls auch Regelungen und Rüstungskontrollvereinbarungen ohne die USA anzustreben. So hätte das Biowaffenüberprüfungsprotokoll auch ohne Washington implementiert werden können. 60 von 61 Staaten waren sich einig. Trotzdem scheiterte die Verabschiedung des Protokolls, weil sich die Amerikaner als einzige Delegation weigerten, dem Papier zuzustimmen. Die Bush-Administration begründete ihre Ablehnung damit, dass das Abkommen nicht verifizierbar sei, obwohl der Vertragsentwurf unter anderem die Einrichtung einer Kontrollbehörde vorsah, die in den Vertragsstaaten Vor-Ort-Inspektionen durchführen sollte.

Auch die amerikanische Seite sollte sich bewusst machen, dass Rüstungskontrolle maßgeblich dazu beigetragen hat, Vertrauen zwischen Ost und West zu schaffen. Sie hat wichtige Anreize geliefert und Ergebnisse gebracht. Gerade in Europa führte sie nicht nur zur Begrenzung oder Abrüstung, sondern sogar zur Vernichtung ganzer Waffenkategorien (INF-Vertrag). Abrüstung und Rüstungskontrolle war und ist ein Grundpfeiler der europäischen Sicherheitsarchitektur. Rüstungskontrolle ist somit kein »überholtes Konzept «, sondern gerade angesichts neuer sicherheitspolitischer Herausforderungen notwendiger denn je. In einem Punkt jedoch hat die amerikanische Seite recht: Die veränderten sicherheitspolitischen Rahmenbedingungen erfordern neue, innovative Konzepte der Verifikation mittels neuer Technologien vor allem im Bereich der Fernerkundung und der Informationstechnologien. M.a.W.: Rüstungskontrolle muss wenn sie eine Zukunft haben soll - in der Lage sein, wirksame Verifikations- und Sanktionsmechanismen zu entwickeln, die auch für die USA glaubwürdig und attraktiv sind.

\section{Die Krise der globalen Nichtverbreitungsregime von Massenvernichtungswaffen}

Spätestens seit dem terroristischen Massenmord vom 11. September steht der mögliche Zugang terroristischer Gruppierungen zu Nuklearmaterial wieder ganz oben auf der Agenda sicherheitspolitischer Überlegungen. Die Konsequenzen illegalen Gebrauchs von Nuklearmaterial und anderen radioaktiven Substanzen stellen eine reale Gefahr für die Menschheit dar. Deshalb ist ein effektives und weltweit flächendeckendes Verifikationsregime zur Kontrolle unautorisierter Nuklearmaterialproliferation unbedingt erforderlich.
So steht mittlerweile eine der größten rüstungskontrollpolitischen Errungenschaften auf dem Spiel: der Atomwaffensperrvertrag (NPT): Er sollte dafür sorgen, dass die nichtnuklearen Staaten auch nichtnuklear blieben. 1995 wurde der NPT zeitlich unbegrenzt verlängert. Tatsächlich aber sind die Tage seiner Wirksamkeit gezählt. Denn längst gibt es eine Zwischenklasse von Staaten, die im System des Sperrvertrages nicht zugelassen ist, die aber dennoch existiert. Israel hat den Vertrag nie unterzeichnet, besitzt um die 200 Atomsprengköpfe in verschiedener Konfiguration und betrachtet diese Waffen als strategische Rückversicherung. Indien führte 1998 erneut eine Kernspaltung durch, Pakistan zog drei Monate später nach. Beide haben um das umstrittene Gebirgsland Kaschmir bislang drei Kriege geführt. Ob die Nuklearwaffen die Führer in Islamabad und Neu Delhi zur Mäßigung zwingen ist mehr eine philosophische als eine strategische Frage. Der Austritt Nordkoreas im Januar 2003 war ein weiterer herber Rückschlag. Nun droht auch Iran mit dem Ausstieg aus dem Atomwaffensperrvertrag. Ausgerechnet die Gralshüter der atomaren Sicherheit, die USA, verstoßen ebenfalls vermehrt gegen den Geist des NPT. Statt sich weiter um die nukleare Abrüstung bspw. im Bereich der nuklearen Kurzstreckenraketen zu kümmern, treibt Washington die Erforschung eigener Atomwaffenprogramme voran. Mini-Nukes spielen bei den Planungen der Bush-Administration und ihren Präventivkriegsszenarien eine zunehmend wichtigere Rolle.

Die sogenannten Schurkenstaaten sehen sich wiederum durch die Pläne der USA und den Dritten Golfkrieg dazu ermutigt, nach der atomaren Waffe als nukleares Faustpfand zu greifen. Das Beispiel Nordkorea zeigt, dass sich Regime, die in das Fadenkreuz der USA geraten sind, nur noch dann sicher wähnen, wenn sie über die Atomwaffe verfügen. Insofern fördert die Bush-Administration geradezu die nukleare Proliferation. Als Präsident Bush von der »Achse des Bösen « sprach, meinte er nicht moralische Verfehlungen, Folter und Unterdrückung. Er nannte Irak, Iran und Nordkorea, weil sie an Nuklearwaffen arbeiten. Tatsächlich ist, weil neue Staaten sich nukleare Macht und dadurch Unverwundbarkeit beschaffen wollen, eine zweite strategische Revolution im Gange. Sie hat das Potenzial, die Welt ins Chaos zu führen, vor allem dann, wenn globaler Terrorismus und Massenvernichtungswaffen in einer Hand zusammenkommen. Iran und Nordkorea sind eine grundlegende Herausforderung für den Fortbestand des Nichtverbreitungsregimes. Statt dem Ziel einer nuklearwaffenfreien Welt droht vielmehr eine »Renuklearisierung « der Weltpolitik.

Der Fall Nordkorea zeigt geradezu exemplarisch einen wunden Punkt des NV-Regimes: Es gibt kaum Instrumente, die Vertragseinhaltung zu erzwingen. Die IAEO hat so gut wie keine Sanktionsmöglichkeiten gegenüber einem Mitglied, das seinen Verpflichtungen nicht nachkommt. Sie ist in einem solchen Fall auf den Sicherheitsrat angewiesen. Die Aktivitäten der IAEO werden zusätzlich behindert durch eine unzureichende finanzielle Ausstattung. Diese ermöglicht es der IAEO nicht, der ihr zugedachten Aufgaben der Verifikation und Sicherheitsgewährung zufriedenstellend auszufüllen. Die Erhöhung der Abrüstungshilfe ist somit dringend erforderlich. Oft sind die Abrüstungskosten die entscheidende Hürde für eine schnelle und komplette Durchführung von Abrüstungsmaßnahmen, beispielsweise bei Landminen oder chemischen 
Waffen. Eine Reihe von Regierungen gewährt derartige Hilfen (v.a. die USA mit dem Nunn-Lugar-Programm), doch sind diese Beträge im Vergleich zu den weltweiten Militärausgaben minimal. Auch stellt sich die Frage, ob eine Trennung zwischen friedlicher und militärischer Nutzung der Kernenergie wirklich möglich ist. Die Aktivitäten der IAEO beschränken sich zudem immer noch nahezu ausschließlich auf die Nichtkernwaffenstaaten, die Mitglied des NVV sind, und lassen die zivilen Brennstoffkreisläufe der Kernwaffenstaaten fast vollständig aus. Für die Verifikation einer kernwaffenfreien Welt müssten nicht nur diese einbezogen, sondern auch noch einmal umfassende Reformen durchgeführt werden, die die Entdeckungswahrscheinlichkeiten weiter erhöhen. Hierbei werden auch Souveränitätsrechte zur Disposition stehen, denn wichtige Maßnahmen wären insbesondere Vor-Ort-Inspektionen mit weitreichenden $\mathrm{Zu}$ gangsrechten und weitere Transparenzmaßnahmen. ${ }^{11}$

Nicht nur die atomaren, sondern auch die biologischen und chemischen Nichtverbreitungsregime befinden sich in einer Krise: Im September 2002 veröffentlichte das IKRK einen eindringlichen Appell, nicht nur an Regierungen, sondern auch an Industrie und Wissenschaft. Um die Verbreitung von mörderischer Biotechnologie $\mathrm{zu}$ verhindern, müssten schnellstens schärfere Gesetze und Kontrollen geschaffen werden, und zwar überall auf der Welt. Einen vergleichbaren Appell des IKRK hat es bisher nur einmal gegeben: 1918, nach den Giftgaseinsätzen im Ersten Weltkrieg. Zwar verbietet seit 1975 das Biowaffen-Übereinkommen die Entwicklung, Herstellung und Lagerung bakteriologischer Waffen und fordert deren Vernichtung. Seit nunmehr zehn Jahren beraten die Mitgliedsländer darüber, wie die Einhaltung des Abkommens in der Praxis kontrolliert werden könnte; gedacht war an eine Lösung nach dem Vorbild der Wiener Atomaufsichtsbehörde. Im November 2001 scheiterten die Verhandlungen endgültig, nicht zuletzt, weil die USA alle Vorschläge zur besseren Kontrolle als aussichtslos verwarfen. Die Supermacht setzt lieber auf verlässliche Eigenvorsorge. Als Teil eines milliardenschweren Terrorschutzprogramms entsteht in Los Alamos gerade ein neues Hochsicherheitslabor, in dem auch an lebenden Erregern geforscht werden kann. Der Rückzug aus der Biowaffenkonvention ist auch auf Druck der US-Industrie zurückzuführen, die befürchtete, dass die ausländischen Kontrolleure durch Industriespionage den Vorsprung der amerikanischen Biotech-Industrie zunichte machen könnten.

Ebenso wie bei den Biowaffen wächst auch die Entwicklung von Chemiewaffen über die völkerrechtlichen Restriktionen hinaus. So werden die meisten neuen Wirkstoffe mittlerweile aus Vorläufern hergestellt, die nicht im CWÜ aufgelistet sind. Genauso besorgniserregend sind die chemischen Altlasten. Das CWÜ, von 147 Staaten ratifiziert, fordert seit 1997 die Vernichtung aller C-Waffen.

Um die Proliferation von Massenvernichtungswaffen wirksam zu bekämpfen, ist ein umfassender internationaler Konsens von Nöten, um die Nichtverbreitungsregime durchzusetzen.

11 Harald Müller/Annette Schaper, Vollständige nukleare Abrüstung Wunschtraum oder konkrete Möglichkeit?, in: Reinhard Mutz/Bruno Schoch/Friedhelm Solms (Hrsg.), Friedensgutachten 1998, Münster/Hamburg 1998, S. 289-302.
Eine wirksame Exportkontrolle durch die Lieferländer ist hierbei mit das wichtigste Instrument, zumal mit wachsender Industrialisierung der Welt auch die Anzahl potenzieller Lieferanten für Dual-use-Technologien zunehmen wird.

\section{Schlussfolgerungen und Empfehlungen}

Für die Fort- und Weiterentwicklung von Abrüstung und Rüstungskontrolle führt das oben Ausgeführte zu einer Reihe von Schlussfolgerungen und Forderungen, mit denen der da nieder liegende Prozess der Rüstungskontrolle wiederbelebt werden sollte. ${ }^{12}$ Dabei muss bedacht werden, dass Rüstungskontrolle und Abrüstung mehr sind, als die Einhegung des Rüstungswettlaufs. Die Vertragsverhandlungen selbst und die Einrichtungen zur Kommunikation und zur Überwachung schaffen die Plattformen für weitere Vertrauens- und Vertragsbildungen. Hierzu gehören:

1. Intensivierung der Abrüstung: Rüstungskontrolle sollte darauf gerichtet sein, Abrüstung zu intensivieren und den Trend zur Abrüstung zu verstetigen. Dies ist besonders dringlich auf dem Gebiet der Nuklearwaffen. Das Versprechen der Nuklearmächte, vollständig nuklear abzurüsten, muss von entsprechenden Taten begleitet werden. Zudem sind - über die 1999 im Rahmen der KSE neu vereinbarten Festlegungen für konventionelle Streitkräfte in Europa hinaus - weitere substanzielle Abrüstungs- und Rüstungskontrollschritte möglich.

2. Verbesserung des Überwachungsregimes der IAEO: $\mathrm{Zu}-$ recht weisen die USA darauf hin, dass das SafeguardSystem der IAEO unzulänglich ist und die vollständige Verifikation der Vertragseinhaltung erfolgreich umgangen werden kann. Hierzu bieten sich mehrere Möglichkeiten an: Zum einen kann man das bestehende System auf der Basis des gültigen Vertragsdokuments reformieren, indem man beispielsweise das vorgesehene, aber bislang ungenutzt gebliebene Instrument der »Sonderinspektionen « aktivieren würde. ${ }^{13}$ Hier böte sich der Iran eventuell als Testfall an. Eine weitere Möglichkeit bestünde darin, das Vertragswerk zu ergänzen und zu reformieren, indem man bspw. Verdachtsinspektionen einführt.

3. Kontrolle von Kleinwaffen und leichten Waffen: Ein besonders vielversprechendes Gebiet der Rüstungskontrolle sind die Bemühungen zur wirksamen Kontrolle der Proliferation von Kleinwaffen und leichten Waffen. Hier sollten die vorhandenen Ansätze im Rahmen der OSZE und der Vereinten Nationen weiter verfolgt und intensiviert werden.

12 Vgl. hierzu auch Michael Brzoska/Herbert Wulf, Krise der Abrüstung, in: Ulrich Ratsch/Reinhard Mutz/Bruno Schoch (Hrsg.), Friedensgutachten 2000, Münster/Hamburg 2000, S. 241-252.

13 Hierbei handelt es sich um Inspektionen, die von der IAEO durchgeführt werden können, wenn dem Safeguard Department Anhaltspunkte für Vertragsverletzungen vorliegen. Die Durchführung einer Sonderinspektion setzt aber das Einverständnis des zu inspizierenden Staates voraus. Falls dieser eine Inspektion ablehnen sollte, ist ein komplizierter Schlichtungsmechanismus zwischen IAEO und der betreffenden Regierung vorgesehen. Als letztes Mittel kann die IAEO schließlich den UN-Sicherheitsrat anrufen. 
4. Durchsetzung selektiver Initiativen: Obwohl es wichtig ist, dass die großen Mächte - und allen voran die USA - sich an Rüstungskontrolle und Abrüstung beteiligen, sollte man Einzelinitiativen gleich gesinnter Staaten nicht von vorn herein ausschließen, wenn die Großmächte die Zusammenarbeit versagen. Es ist nicht ungewöhnlich, dass die Unterschriften wichtiger Regierungen unter Rüstungskontrollverträgen fehlen. Die Landminen-Konvention ist hier keine Ausnahme und macht deutlich, dass auch selektive Initiativen Fortschritte erreichen können.

5. Finanzielle und technische Abrüstungshilfe: Die Erhöhung der Abrüstungshilfe ist dringend erforderlich. Oft sind die Abrüstungskosten die entscheidende Hürde für eine schnelle und komplette Durchführung von Abrüstungsmaßnahmen, beispielsweise bei Landminen oder chemischen Waffen. Eine Reihe von Regierungen gewährt derartige Hilfen (v.a. die USA mit dem Nunn-LugarProgramm), doch sind diese Beträge im Vergleich zu den weltweiten Militärausgaben minimal. Die Globale G8-Partnerschaft gegen die Verbreitung von Massenvernichtungswaffen und deren Bestätigung auf dem Weltwirtschaftsgipfel von Evian, sind hier uneingeschränkt zu begrüßen.

6. Gegenüber der Gefahr chemischer und biologischer Waffen kommt es darauf an, ein dichtes Netz von Transparenzmaßnahmen zu entwickeln, die eine Früherkennung entsprechender Aktivitäten erlauben und der Staatengemeinschaft so die Chance zum rechtzeitigen Handeln geben. Die bestehenden Verträge sollten zügig und unter Einbeziehung wirksamer Verifikationsmaßnahmen ausgebaut werden.

7. Im Bereich der nuklearen Abrüstung sollten folgende Maßnahmen angestrebt werden: Ein umfassender Teststopp mit lückenloser Verifikation, die Erweiterung der $\mathrm{Zu}$ gangsrechte der IAEO-Waffeninspektoren in allen Nichtkernwaffenstaaten, ein START-III-Abkommen, das die Arsenale der kleineren Kernwaffenstaaten mit einbeziehen und strikter Verifikation unterwerfen müsste, sowie ein UN-Kernwaffenregister. Im Rahmen der NATO sollte die Frage der Abrüstung der nuklearen Kurzstreckenraketen wieder auf die Tagesordnung gesetzt werden.

8. Wirksame Kontrolle von Trägertechnologien: Entwicklung, Erwerb, Besitz und Weitergabe von militärischer Trägertechnologie sind bislang nicht durch völkerrechtliche Verbots- bzw. Nichtverbreitungsnormen geregelt und auch das Exportkontrollregime »Missile Technology Control Regime (MTCR) ist in seiner Wirksamkeit beschränkt. Die Raketenproliferation hat in den letzten Jahren erheblich zugenommen und birgt ernsthafte Risiken für die Stabilität und Sicherheit der betroffenen Regionen. Mit der Unterzeichnung des »Haager Verhaltenskodex gegen die Proliferation ballistischer Raketen « am 25. November 2002 wurde ein erster Schritt unternommen, um diese Lücke zu schließen.

9. Im OSZE-Rahmen bietet sich die Einrichtung einer OSZEAgentur für Rüstungskontrolle und Abrüstung an, die aus dem Konfliktverhütungszentrum hervorgehen könnte.
10. Ziel europäischer Politik sollte eine wirksame Verknüpfung von Abrüstung und Nichtweiterverbreitung sein. Abrüstung muss zum Instrument der Bekämpfung der Verbreitung von MVW werden. Diese wiederum ist die Voraussetzung für weitere Abrüstung. Multilaterale Verträge und wirksamere Exportkontrollregime sollten als Teil einer gemeinsamen Strategie weiterentwickelt werden. Die transatlantische Erklärung vom 26. Juni 2003 geht in die richtige Richtung. Darin erkennen die Vereinigten Staaten die Bedeutung multilateraler Verträge an und erklären sich zumindest deklaratorisch zu einer gemeinsamen Strategie im Kampf gegen die Weitergabe von Massenvernichtungswaffen bereit.

11. Entscheidend bleibt jedoch eine verbesserte Verifikation. Nur so wird man die Vereinigten Staaten mittel- bis langfristig wieder von der Sinnhaftigkeit internationaler Rüstungskontrolle überzeugen können. Verifikation braucht »Zähne« und Phantasie, um wirksam zu werden. Hierzu gehören unangemeldete Vor-Ort-Inspektionen, der Einsatz neuer Überwachungstechnologien und der Aufbau von qualifizierten unparteiischen Inspektorenteams, auf die im Bedarfsfall schnell und unbürokratisch zugegriffen werden können muss. Nach Vorbild der Stand-by Forces für Peacekeeping-Operationen, wäre auch der Aufbau von kompetenten Stand-by-Inspektionsteams in Betracht zu ziehen.

\section{Fazit}

Der bilaterale Rüstungskontrollansatz des Kalten Krieges ist obsolet geworden. Neue multilaterale Ansätze müssen gefunden werden, um die unkontrollierte Weiterverbreitung von neuen Rüstungstechnologien zu regeln und $\mathrm{zu}$ begrenzen. Rüstungskontrolle wandelt sich zunehmend von einem erfolgsorientierten zu einem prozessorientierten Ansatz. Probleme auf diesem dornigen Weg sind die unterschiedlichen Militärpotenziale und Einsatzdoktrinen, die verschiedenen Rechtskulturen und die fortschreitende Weiterverbreitung. Angesichts der rüstungstechnischen und militärischen Überlegenheit der USA und dem augenscheinlichen Desinteresse der augenblicklichen Administration, wird Rüstungskontrolle immer schwieriger umsetzbar. Nicht zuletzt aus diesem Grunde ist die Stabilisierung essenzieller Rüstungsregime in Europa (KSE) wie weltweit (NPT) dringend geboten. Eine vorausschauende Behandlung impliziert auch, vorhandene Verträge »krisenfest « zu machen. Eine Betonung kooperativer Sicherheit an den Grenzen Europas im Hinblick auf den Nahen/Mittleren Osten und Asien erscheint ebenso geboten zu sein, wie die Erarbeitung neuer Initiativen im Bereich neuer Technologien und Kleinwaffen. Regionale Initiativen haben dabei nicht nur den Vorteil, den Rüstungswettlauf zu bearbeiten, sie erzielen in der Regel auch einen Spin-off-Effekt, da die Gespräche, Ergebnisse und Verfahren zumeist der Beginn für Vertrauens- und weitere Regimebildungen sind. Darüber hinaus muss die Rüstungskontrolle der Zukunft den neuen asymmetrischen Kriegsbildern (Terrorismus, Bürgerkriege, Low Intensity Warfare etc.) angepasst werden. Insgesamt lässt sich festhalten, dass die Rüstungskontrolle vor dem Hintergrund weiterhin enormer Rüstungsstände in Europa, der verstärkten technologischen Dynamik und internationaler 
Technologiekonkurrenz, des sich ausweitenden Welthandels, der fortschreitenden militärtechnologischen Revolution, ungelöster regionaler Konflikte und fehlender Konfliktlösungsmechanismen vor völlig neuen Herausforderungen steht. Dabei müssen sowohl die Erfolge der bisherigen Rüstungskontrollbemühungen gegen machtpolitische Alleingänge der USA gesichert werden, als auch die neuen Herausforderungen durch die zunehmende Kommerzialisierung und Privatisierung von Rüstung und Sicherheit bewältigt werden.

Internationale und regionale Abkommen zur Kontrolle und Abrüstung der vorhandenen Waffenarsenale verbunden mit der Einhaltung und gegebenenfalls Verschärfung von Rüstungsexportrichtlinien bleiben unverzichtbare Instrumente, um der Verbreitung von Massenvernichtungswaffen und konventionellen Waffen aller Art Einhalt zu gebieten. Die Behauptung, die klassischen Instrumente der Rüstungskontrolle funktionierten nicht mehr, ist ein wohlfeiles Argument, das in Washington bereits Anfang der neunziger Jahre formuliert wurde: Rüstungskontrolle und Verifikation wird es niemals flächendeckend und allumfassend geben. Auch künftig wird es Möglichkeiten geben, Rüstungskontrollverträge und die darin enthaltenen Kontroll- und Überprüfungsmechanismen zu umgehen bzw. zu unterlaufen. Gleichwohl gibt es zur vertragsbasierten und verifizierbaren Rüstungskontrolle nur eine Alternative: ein weltweites nukleares, chemisches und biologisches Wettrüsten. Ein solches kann auch nicht im Interesse der USA liegen. Die Risiken, die aus der Trias der Bedrohung durch transnationalen Terrorismus, Massenvernichtungswaffen und zerfallende Staaten resultieren, sind jedenfalls mit nachrichtendienstlichen, rüstungskontrollpolitischen und polizeilichen Instrumentarien wirksamer $\mathrm{zu}$ bekämpfen als durch militärische Interventionen.

\section{Rüstungskontrolle in Asien: Regionale Konzepte und ihre Erfolgsbedingungen}

\begin{abstract}
Dr. Christopher Daase, Lecturer in International Relations, University of Kent at Canterbury, Brussels School of International Studies, Brüssel/ Prof. Dr. Susanne Feske, Institut für Politikwissenschaft, Westfälische Wilhelms-Universität Münster.
\end{abstract}

terogenität Asiens, die das Problem der Rüstungsdynamik ungleich komplexer macht als dies in Europa oder generell in der nördlichen Hemisphäre der Fall ist. Genauso wichtig ist allerdings die Abneigung gegen Konzepte, die als vom Westen den asiatischen Staaten aufgezwungen empfunden werden: Was für Europa richtig war oder ist, muss für Asien nicht auch richtig sein. ${ }^{1}$ Diese Empfindlichkeit ist Ausdruck historisch begründeter Erfahrungen während der Kolonialzeit; aber auch in der Gegenwart wird von asiatischer Seite immer wieder argumentiert, dass der sich als überlegen fühlende Westen weiterhin versucht, die südliche Hemisphäre zu dominieren und ihr das eigene Werte- und Normensystem und letztlich auch die eigenen Problemlösungsstrategien überzustülpen. Drittens werden auch kulturelle Argumente ins Feld geführt, die erklären sollen, warum westliche Rüstungskontrollkonzepte für Asien ungeeignet sind. Die Verhandlungs- und Kooperationskultur in Asien weist nämlich Besonderheiten auf, die in westlichen Konzepten keine Rolle spielen. Ein zirkuläres Zeitverständnis in Asien führt beispielsweise dazu,

1 Vgl. Gill Bates/J.M. Mak, Introduction, in: Dies., (Hrsg.), Arms, Transparency and Security in Southeast Asia, Oxford 1997, 1-9, 2. dass Tagesordnungen oder Verhandlungsabläufe anders organisiert werden als in westlichen Modellen, denen ein lineares Zeitverständnis zugrundeliegt. Außerdem spielt in Asien der Konsensgedanke eine wichtige Rolle. Es geht deshalb nicht in erster Linie darum, die eigene bargaining power zu erhöhen und Maximalforderungen durchzusetzen. Ein gutes Ergebnis aus asiatischer Sicht berücksichtigt vielmehr die legitimen Interessen aller Beteiligten und sollte diese miteinander in Einklang bringen. In der Praxis bedeutet dies, dass Verhandlungen weniger ergebnisorientiert geführt und Erfolge nicht unbedingt Schritt für Schritt als chronologischer Fortschritt erzielt werden. Das Ziel von Verhandlungen ist nicht notwendigerweise eine schriftliche Vereinbarung, die unterschrieben und ratifiziert wird. Verhandlungsprozesse haben in Asien durchaus einen Selbstzweck, insofern sie die Kommunikation zwischen Antagonisten ermöglichen und das harmonische Aushandeln und Abgleichen von Postitionen gestatten. Auch hierin unterscheidet sich die westliche Vertragskultur von der asiatischen Verhandlungskultur.

Angesichts dieses Widerwillens, auf Theorien und Konzepte der Rüstungskontrolle zurückzugreifen, die während des Kalten Krieges in Europa und den Vereinigten Staaten entwickelt worden sind, ${ }^{2}$ ist es notwendig, nach indigenen Ansätzen und Ideen kooperativer Rüstungssteuerung in Asien zu suchen

2 Vgl. dazu insbesondere Jozef Goldblat, Arms Control: A Guide to Negotiations and Agreements, London 1994; Stuart Croft, Strategies of Arms Control: A History and Typology, Manchester 1996; Michael O. Wheeler, A History of Arms Control, in: Jeffrey A. Larsen, (Hrsg.), Arms Control. Cooperative Security in a Changing Environment, Boulder 2002, 19-39. 